. NUREG/CR-0240

BNL-NUREG-50859

\title{
A SINGLE-PHASE PUMP MODEL FOR ANALYSIS OF LMFBR HEAT TRANSPORT SYSTEMS
}

\author{
Imtiaz K. Madni and Erik Cazzoli
}

Date Published - June 1978

\author{
ENGINEERING AND ADVANCED REACTOR SAFETY DIVISION \\ DEPARTMENT OF NUCLEAR ENERGY, BROOKHAVEN NATIONAL LABORATORY \\ UPTON, NEW YORK 11973
}




\section{DISCLAIMER}

This report was prepared as an account of work sponsored by an agency of the United States Government. Neither the United States Government nor any agency Thereof, nor any of their employees, makes any warranty, express or implied, or assumes any legal liability or responsibility for the accuracy, completeness, or usefulness of any information, apparatus, product, or process disclosed, or represents that its use would not infringe privately owned rights. Reference herein to any specific commercial product, process, or service by trade name, trademark, manufacturer, or otherwise does not necessarily constitute or imply its endorsement, recommendation, or favoring by the United States Government or any agency thereof. The views and opinions of authors expressed herein do not necessarily state or reflect those of the United States Government or any agency thereof. 


\section{DISCLAIMER}

Portions of this document may be illegible in electronic image products. Images are produced from the best available original document. 


\title{
A SINGLE-PHASE PUMP MODEL FOR ANALYSIS OF LMFBR HEAT TRANSPORT SYSTEMS
}

\author{
Imtiaz K. Madni and Erik Cazzoli*
}

\author{
Manuscript Completed - May 1978 \\ Date Published - June 1978
}

*Applied Mathematics Department, BNL

\section{ENGINEERING AND ADVANCED REACTOR SAFETY DIVISION \\ DEPARTMENT OF NUCLEAR ENERGY, BROOKHAVEN NATIONAL LABORATORY UPTON, NEW YORK 11973}

Prepared for the

UNITED STATES NUCLEAR REGULATORY COMMISSION

OFFICE OF NUCLEAR REGULATORY RESEARCH

Contract No. EY-76-C-02-0016 
NOTICE

This report was prepared as an account of work sponsored by an agency of the United States Government. Neither the United States Government nor any agency thereof, or any of their employees, makes any warranty, expressed or implied, or assumes any legal liability or responsibility for any third party's use, or the results of such use, of any information, apparatus, product or process disclosed in this report, or represents that its use by such third party would not infringe privately owned rights.

Available from

U.S. Nuclear Regulatory Commission

Washington, D.C. 20555

Available from

National Technical Information Service

Springfield, Virginia 22161 


\section{TABLE OF CONTENTS}

Page

FOREWORD ..................... . iv LIST OF FIGURES ....................... v

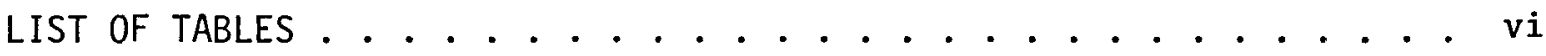

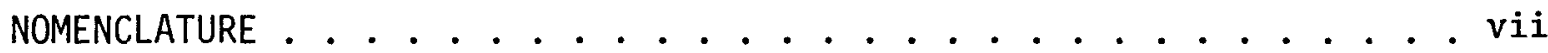

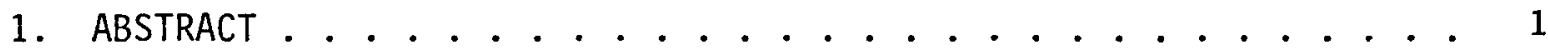

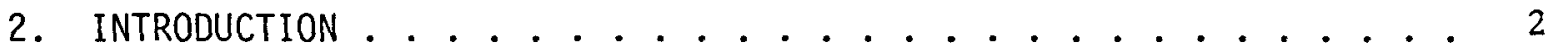

3. ANALYSIS ................... 4

3.1 IMPELLER DYNAMICS .................... 4

3.1.1 Homologous Characteristics ........... 6

3.1.2 Pump Speed Equation ............ 13

3.2 PUMP TANK (RESERVOIR) . . . . . . . . . . . . 14

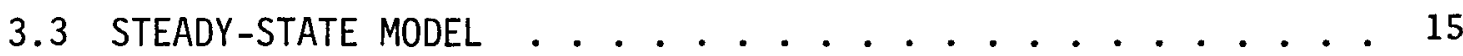

3.4 PONY MOTOR OPTION . . . . . . . . . . . . 17

3.5 SYSTEM THERMAL-HYDRAULICS . . . . . . . . . 17

4. SAMPLE RESULTS ............... 20

4.1 PUMP COASTDOWN WITH REACTOR SCRAM .......... 20

4.2 DOUBLE-ENDED PIPE BREAK IN THE PHTS . . . . . . . . 29

5. DISCUSSION ................. 36 APPENDIX A

A.1 PUMP PERFORMANCE CURVES ............ 38

A.2 PUMP FRICTION . . . . . . . . . . 42 REFERENCES .................. . . 43 


\section{FOREWORD}

As a part of the Super System Code (SSC) development project for simulating thermohydraulic transients in LMFBRs, a single-phase model for the centrifugal pumps in the primary and intermediate heat transport systems was developed. This topical report describes the model in detail and presents results of some test cases for a loop-type LMFBR.

This work, covered under the budget activity No, 60-19-20-01-1, was performed for the office of the Assistant Director for Advanced Reactor Safety Research, Division of Reactor Safety Research, United States Nuclear Regulatory Commission. 


\section{LIST OF FIGURES}

Figure No.
Title

Page

Schematic diagram for the pump mode 1

5

Pump configurations under different regimes of operation

7

Complete homologous head curves $\quad 10$

Complete homologous torque curves

11

Primary system configuration for two-loop simulation

18

Computed primary pump speed decay for Case 1 : pump coastdown with reactor scram

Computed pump-to-vessel flow decay for Case 1

24

Computed decay of primary pump head for Case 1

Transient operating points on pump head curves for Case 1

Comparison of predicted pump speed and flow decay for Case 1 using DEMO and RELAP3B pump friction equations

Comparison of predicted speed and flow transients for Case 1 using pump characteristics from DEMO and present model

Computed pump flow, speed and head transients in broken and intact loops for Case 2: double-ended pipe break in the PHTS

Transient operating points on homologous head curves for broken loop pump

Transient operating points on homologous head curves for intact loop pump 


\section{LIST OF TABLES}

Table I

A. I

A. I I
Title

Primary pump design data used in the analysis Head polynomial coefficients

Torque polynomial coefficients
Page

21

40

41 


\section{NOMENCLATURE}

Symbol

A

g

$\mathrm{h}$

H

I

K

$\mathrm{L}$

m

N

$\mathrm{P}$

$P_{1}$

$P_{\text {inV }}$

Q

$\mathrm{R}_{\text {gas }}$

t

T

W

W

l, 1

$W_{2,1}$

$W_{3,1}$

$W_{1,2}$

$W_{2,2}$

$\mathrm{Z}_{\mathrm{R}}$
Description

Flow or cross-sectional area

Acceleration due to gravity

Normalized pump head $\left(H / H_{R}\right)$

Pump head

Moment of inertia

Loss coefficient

Length of pipe section

Mass

Pump speed

Pressure

Inlet pressure to loop

Pressure at reactor vessel inlet

Pump volumetric discharge

Gas constant

Time

Temperature

Mass flow rate

Flow rate into broken loop from reactor vessel

Flow rate downstream of pump in broken loop

Flow rate downstream of break

Vessel-to-pump flow rate, intact loop

Pump-to-vessel flow rate, intact loop

Level of coolant in reservoir
Units

$\mathrm{m}^{2}$

$\mathrm{m} / \mathrm{s}^{2}$

m

$\mathrm{kg}-\mathrm{m}^{2}$

-

m

$\mathrm{kg}$

RPM

$\mathrm{N} / \mathrm{m}^{2}(\mathrm{~Pa})$

$\mathrm{N} / \mathrm{m}^{2}$

$\mathrm{N} / \mathrm{m}^{2}$

$\mathrm{m}^{3} / \mathrm{s}$

$\mathrm{m}^{2} / \mathrm{s}^{2} \mathrm{k}$

$\mathrm{S}$

K

$\mathrm{kg} / \mathrm{s}$

$\mathrm{kg} / \mathrm{s}$

$\mathrm{kg} / \mathrm{s}$

$\mathrm{kg} / \mathrm{s}$

$\mathrm{kg} / \mathrm{s}$

$\mathrm{kg} / \mathrm{s}$

m 


\section{NOMENCLATURE (Cont)}

Symbol

$\mathrm{Z}_{\mathrm{RV}}$

$z_{\text {tot }}$

$\Delta \mathrm{P}$

$\Delta \mathrm{P}_{f, g}$

$\alpha$

$\beta$

$v$

$\rho$

$\tau$

$\Omega$

Description

Level of coolant in upper plenum

Height of reservoir

Pressure loss (or drop)

Sum of pressure losses due to friction, gravity, etc. $\mathrm{N} / \mathrm{m}^{2}$

Normalized pump speed $\left(\Omega / \Omega_{R}\right)$

Normalized hydraulic torque $\left(\tau_{\text {hyd }} / \tau_{R}\right)$

Normalized pump discharge $\left(Q / Q_{R}\right)$

Coolant density

Torque

Pump speed

Subscript

acc

c

$\mathrm{CV}$

EV

fr

g

gas

hyd

IHX, S

in

j
Acceleration

Core

Check valve

Evaporator

Friction

Gravity

Value for cover gas in reservoir

Hydraul ic

IHX, secondary side

Inlet (usually, to pump)

Pipe number in loop
Units

m

m

$\mathrm{N} / \mathrm{m}^{2}$

$-$

$-$

$-$

$\mathrm{kg} / \mathrm{m}^{3}$

$N-m$

$\mathrm{rad} / \mathrm{s}$ 
NOMENCLATURE (Cont)

Subscript

Description

Units

m

out

$\mathrm{R}$

res

RV

SH

1

2

Motor

Outlet (usually, from pump)

Rated value

Reservoir

Reactor vessel

Superheater

Broken loop

Intact loop 


\section{ABSTRACT}

A single-phase pump model for transient and steady-state analysis of LMFBR heat transport systems is presented. Fundamental equations of the model are angular momentum balance to determine transient impeller speed and mass balance (including thermal expansion effects) to determine the level of sodium in the pump tank. Pump characteristics are modeled by homologous head and torque relations. All regions of pump operation are represented with reverse rotation allowed. The model also includes option for enthalpy rise calculations and pony motor operation. During steady state, the pump operating speed is determined by matching required head with total load in the circuit. Calculated transient results are presented for pump coastdown and double-ended pipe break accidents. The report examines the influence of frictional torque and specific speed on predicted response for the pump coastdown to natural circulation transient. The results for a double-ended pipe break accident indicate the necessity of including all regions of operation for pump characteristics. 


\section{INTRODUCTION}

A major concern in the safety analysis of LMFBR systems is whether sufficient cooling capability is provided to keep fuel element clad temperatures below specified values during off-normal and accident events that may be postulated to occur in the heat transport system, e.g., loss of all pumping power in an intact system, or a loss-of-piping-integrity accident, with reactor scram. The behavior of the centrifugal pumps which circulate the reactor coolant becomes extremely important during such transients, and the ability to predict pump performance is required to understand and predict the interrelated hydraulic phenomena controlling loop and core flow rates.

As an example, the loss-of-piping-integrity accident starts when a rupture occurs in the primary piping and with coolant leaking out of the break and pumps coasting down, the system loses driving pressure for flow through the core. To answer the concern regarding core coolability, it is necessary to know how the pumps perform in both the broken and unbroken loops of the primary system. The conditions imposed on the pump are determined by the type of accident postulated (location and size of rupture), pump location (whether in broken or unbroken 10op), and the thermal-hydraulic response of the rest of the system. The flow through the pump may completely reverse direction, as can the rotation, causing the pump to go through several regimes of operation. Therefore, analys is is required that takes into account the changes occurring in the characteristics as the speed and flow change.

In this report, a single-phase model for the LMFBR sodium pump will be des-

cribed, as it has been developed for the Super system Code. (1) The model allows reverse rotation, covers all regions of pump operation while representing pump characteristics as homologous relations, computes pump enthalpy rise, includes 
thermal expansion effects of coolant in the pump tank, and has pony motor option. The model equations are solved together with system equations to anaTyze the pump behavior and account for the interaction between the impeller and the flow resistance in the circuit, during both steady state and off-normal or accident conditions.

Results of calculated conditions for pump coastdown and double-ended pipe break accidents are presented in Chapter 4. For the case of double-ended pipe break, results for both broken and intact loop pumps are shown. 


\section{ANALYSIS}

Liquid sodium pumps for LMFBRs are vertically mounted, free surface, centrifugal pumps driven by a variable speed motor. The impeller is attached to the bottom of a shaft. The shaft is surrounded by a tank which extends upward from the pump casing to the motor mounting. The level of sodium in this tank is maintained well below the bearings which reside in an atmosphere of inert gas such as argon. (2)

The primary purpose of the analytical pump model is to calculate the pressure rise across the pump for use in the loop hydrautic analysis. Since the pressure rise, or head, is dependent on pump speed, the speed is calculated throughout the transient. This yields a variable pump head, dependent on transient conditions.

The sodium tank essentially sees only the inlet pressure to the pump at its lower end, and the cover gas pressure acting on the sodium free surface at its upper end. Hence, for modeling purposes, the pump is most conveniently divided so that a free-surface pump tank is located just upstream of the pump impeller (see Figure 1). The impeller is modeled in terms of homologous head and torque relations which describe pump characteristics, and angular momentum balance to determine transient impeller speed. The behavior of the free surface in the pump tank is described by a mass balance at the tank.

\subsection{IMPELLER DYNAMICS}

The model is based on the following simplifying assumptions:

1) The impeller is a single control volume.

2) Flow rate in is equal to flow rate out (i.e., no accumulation of mass in the impeller control volume). 


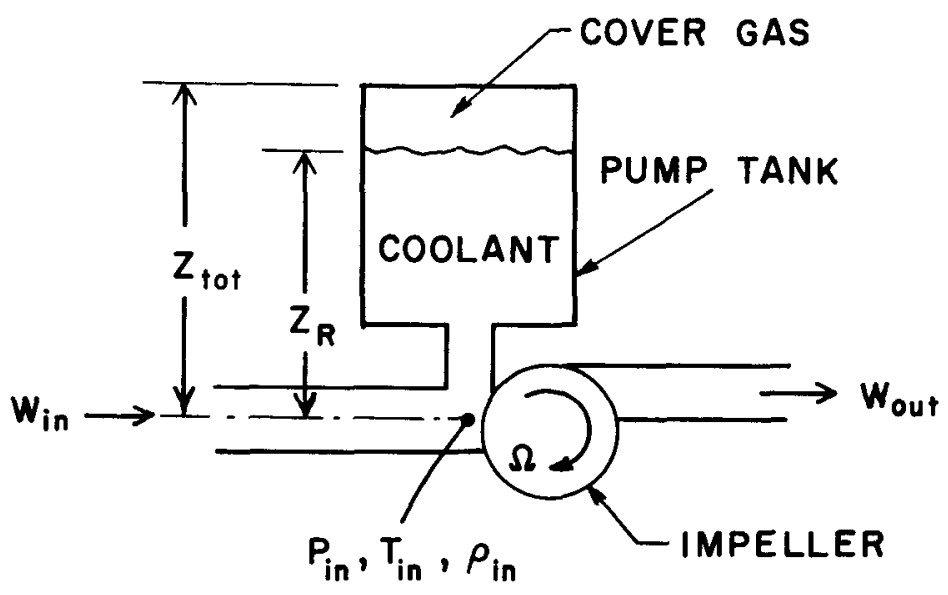

Figure 1. Schematic diagram for the pump mode1. 
3) Pump characteristics, based on steady state data, are applicable during transient operation. This assumption implies that the instantaneous performance of the machine at any time during a transient is identical with the steady state performance for the same operating conditions.

4) Scale effects are considered negligible.

5) Enthalpy rise is computed based on quasi-steady process with no storage terms.

6) Single-phase model (no cavitation effects).

\subsubsection{Homologous Characteristics}

In a severe accident such as a double-ended pipe break in the cold leg of a primary heat transport 10op, with power to pumps being interrupted, the pump can go through several regimes of operation. These are illustrated in Figure 2. In the initial stages of the transient, with positive rotational speed and positive discharge, the pump is operating in the zone of normal operation. As flow reverses in the loop, the pump enters the zone of energy dissipation, unti1 pump speed also reverses. With both flow and speed negative, the pump is in the zone of turbine operation. Much later, if the flow recovers and becomes positive again, the pump enters the zone of reverse pump operation. Pump performance curves encompassing all these regions of operation constitute the complete characteristics of the pump.

Single-phase pump performance is generally measured and described in terms of head and torque (hydraulic, friction, and/or total) for a given speed, volume flow rate, and fluid density. These are the pump characteristic parameters. For a proper simulation of the pump behavior, we need values of these parameters for al1 regions of interest, and for the full-scale pump in question. However, pump 


$$
\begin{aligned}
& \nu=Q / Q_{R} \\
& a=\Omega / \Omega_{R}
\end{aligned}
$$

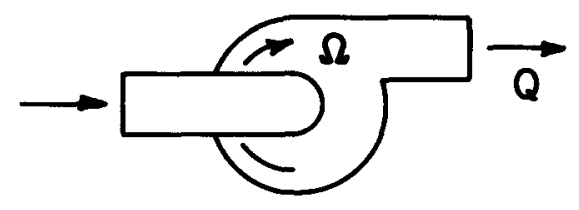

$$
\begin{aligned}
& \text { NORMAL PUMP } \\
& (+\nu,+\alpha)
\end{aligned}
$$

\section{ENERGY DISSIP. \\ $(-\nu,+a)$}

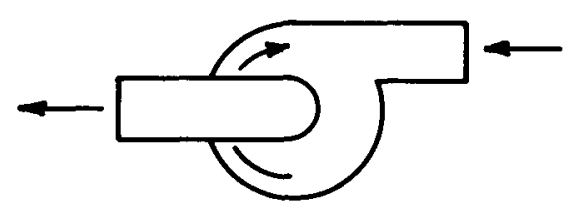

NORMAL TURBINE

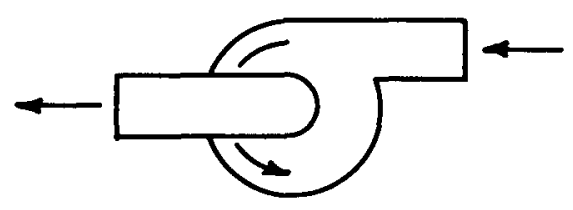

$(-\nu,-\alpha)$

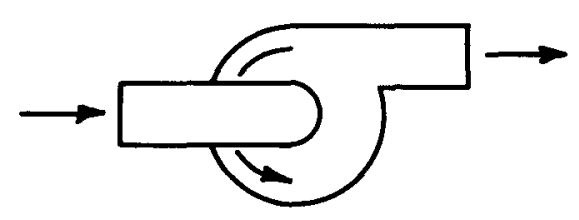

\section{REVERSE PUMP}

$(+\nu,-a)$

Figure 2. Pump configurations under different regimes of operation. 
manufacturers generally supply performance data of their units for normal operation only. Due to practical limitations, most additional data available is from scaled-down models, not necessarily with liquid metal, and from selected combinations of operating conditions within the capabilities of the test facility. Hence, to obtain the complete characteristics, we resort to homologous theory, which enables us to use the results of model tests with similar pumps, and also to extend the selected results to cover all possible combinations of pump parameters. The parameter defining the similarity of the pumps is the "specific speed," defined as

$$
N_{S}=\frac{N \sqrt{Q}}{H^{3 / 4}}
$$

where

$$
\begin{aligned}
& N=\text { speed }(\mathrm{RPM}) \\
& Q=\text { flow rate }(\mathrm{gPm}) \\
& \mathrm{H}=\text { head rise }(\mathrm{ft} .)
\end{aligned}
$$

A specific speed can be calculated for any given operating condition of a pump. However, the specific speed that gives maximum efficiency for a particular unit identifies the pump type related to the impeller design.

If the pump parameters are non-dimensionalized through division by the appropriate rated values, then the non-dimensional (homologous) characteristics of the pump are independent of the liquid pumped and the shape of the characteristic curves then depends only on the rated specific speed. $(4,5)$ This assumes negligible viscosity effects. In general, the influence of viscosity and other scale effects on pump head and torque is small for single-phase flow. (6)

If pump head, torque, flow rate and speed are divided by their respective rated values, the dimensionless parameters are written as follows: 


$$
\begin{aligned}
& h=H / H_{R} \\
& \beta=\tau_{\text {hyd }} / \tau_{R} \\
& \nu=Q / Q_{R} \\
& \alpha=\Omega / \Omega_{R}
\end{aligned}
$$

Homologous head curves can then be drawn by plotting

$$
\frac{h}{\alpha^{2}} \text { vs } \frac{\nu}{\alpha} \quad \text { in the range } \quad 0 \leq\left|\frac{\nu}{\alpha}\right| \leq 1
$$

and

$$
\frac{h}{v^{2}} \text { vs } \frac{\alpha}{v} \quad \text { in the range } \quad 0 \leq\left|\frac{\alpha}{v}\right|<1
$$

Since both $\alpha$ and $v$ pass through zero during the course of a pump reversal, it is necessary to use both $h / \alpha^{2}$ vs $v / \alpha$ and $h / v^{2}$ vs $\alpha / v$ to avoid having the curves go to infinity.

Similarly, torque curves can be drawn by plotting

$$
\frac{\beta}{\alpha^{2}} \text { vs } \frac{v}{\alpha} \quad \text { in the range } \quad 0 \leq\left|\frac{v}{\alpha}\right| \leq 1
$$

and

$$
\frac{\beta}{v^{2}} \text { vs } \frac{\alpha}{v} \quad \text { in the range } \quad 0 \leq\left|\frac{\alpha}{\nu}\right|<1
$$

Figures 3 and 4 show these curves encompassing al1 four quadrants and al1 regions of pump operation. Three letters are used for each curve segment; first, $B$ or $\mathrm{H}$ to designate torque or head ratio, second $A$ or $V$ to designate division by 


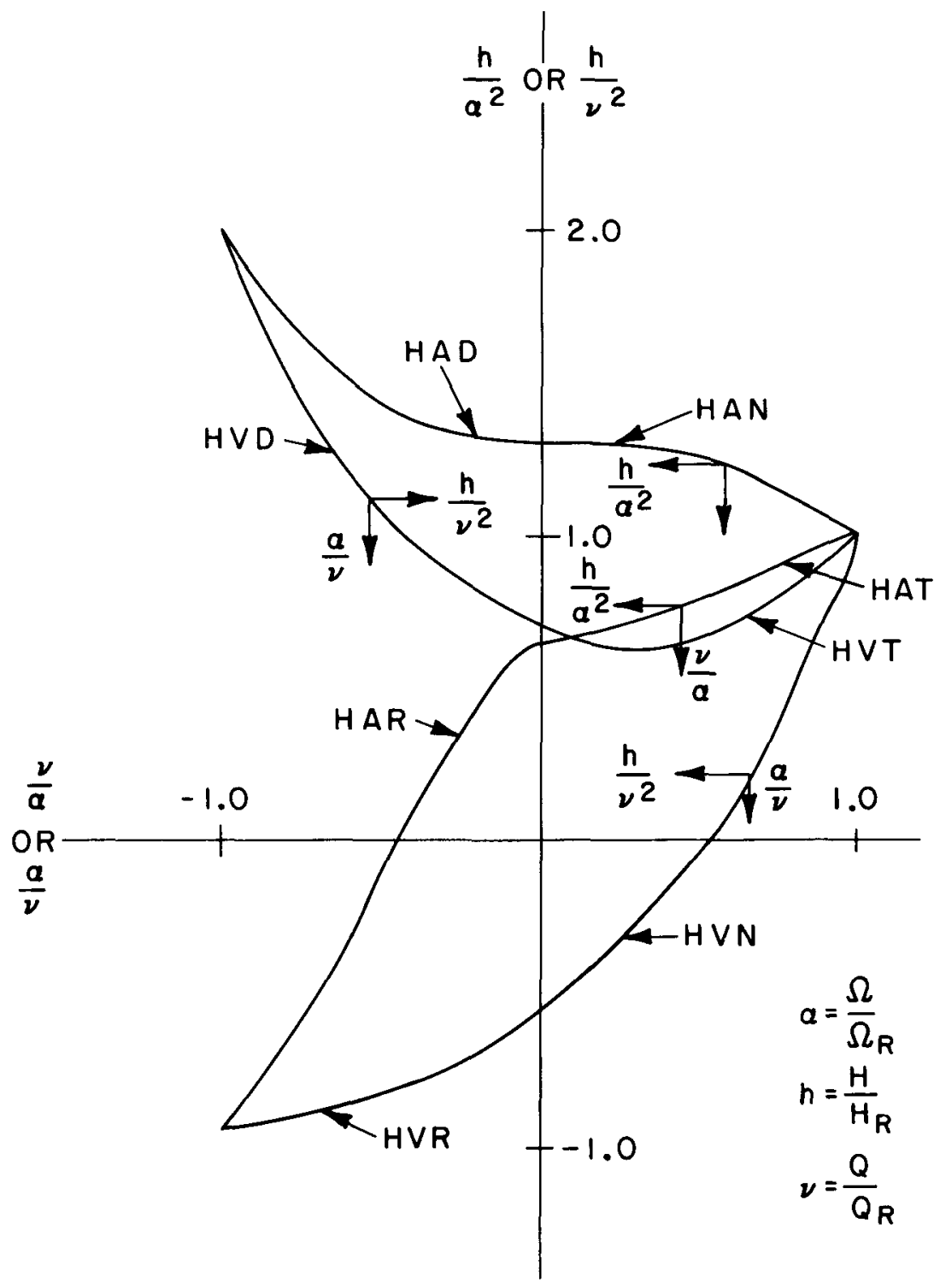

Figure 3. Complete homologous head curves. 


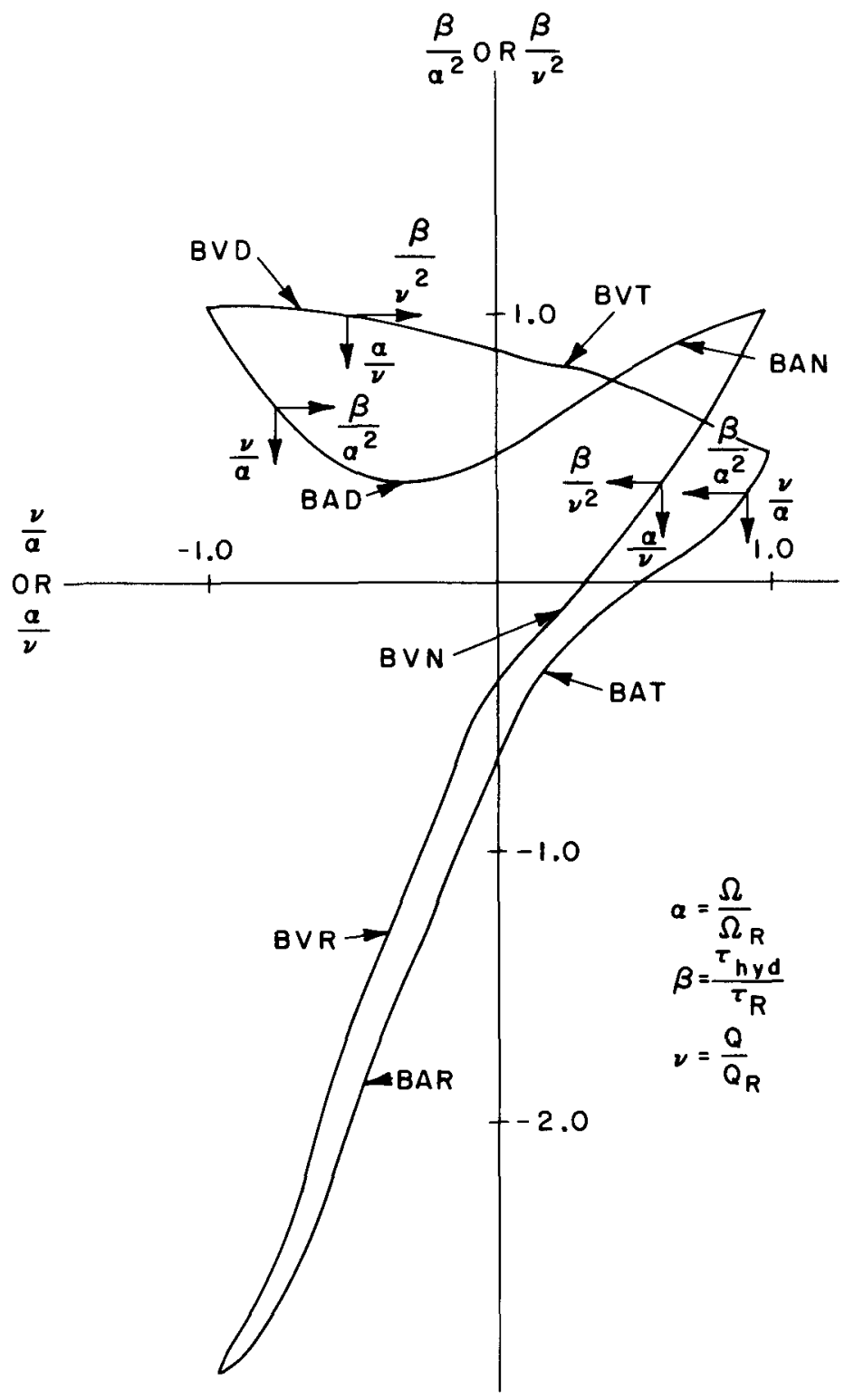

Figure 4. Complete homologous torque curves. 
$\alpha^{2}$ or $\nu^{2}$; third N, D, T or R to indicate normal, energy dissipation, turbine, or reverse pump operation zone. The curves can either be read in tabular form, as is done in the RELAP3B code, ${ }^{(7)}$ or in the form of curve-fitted polynomials. In this analysis, the latter approach has been used. The polynomial relations are of the following form (up to 5 th order):

$$
\begin{array}{r}
\frac{\beta}{\alpha^{2}} \text { or } \frac{h}{\alpha^{2}}=c_{1}+c_{2} \frac{\nu}{\alpha}+c_{3}\left(\frac{v}{\alpha}\right)^{2}+\ldots+c_{6}\left(\frac{v}{\alpha}\right)^{5} \\
\text { in the range } 0 \leq\left|\frac{v}{\alpha}\right| \leq 1 \\
\frac{\beta}{v^{2}} \text { or } \frac{h}{v^{2}}=c_{1}+c_{2} \frac{\alpha}{v}+c_{3}\left(\frac{\alpha}{v}\right)^{2}+\ldots+c_{6}\left(\frac{\alpha}{v}\right)^{5} \\
\text { in the range } 0 \leq\left|\frac{\alpha}{v}\right|<1
\end{array}
$$

where $c_{1}, c_{2}, \ldots c_{6}$ are user-specified constants for each polynomial. There are seven polynomials for head-discharge and seven for torque-discharge. This is a very convenient representation and avoids table-lookups during computation. The SSC code has built in coefficients for a pump with specific speed 1800 (gpm units). This is in the range of specific speeds for LMFBR pumps (e.g., for FFTF, $N_{S} \simeq 1400$, for Phenix, $N_{S} \simeq 2000$, and for $C R B R, N_{S} \simeq 2200$ ). However, if the user would prefer to use vendor-supplied data wherever possible, some or all of the built-in coefficients can be overridden. The coefficients have been obtained by curvefitting the data points in Streeter ${ }^{(3)}$ for all regions except reverse pump, for which the values are not available. The reverse pump region connects turbine operation zone to normal operation zone and corresponds to positive flow and negative impeller rotational speed (see Figure 2). This condition can occur during the later stages of the transient following pipe break (without pony motor). Values for this regime were generated from the Karman Knapp Circle diagram for 
$N_{S}=1800^{(4)}$ and then curve-fitted. More details on pump coefficients are provided in Appendix A.

Once the characteristics are available in this fashion, the transient head $H$ and torque $\tau_{\text {hyd }}$ can be determined, knowing rated values, operating speed and flow rate. The head then yields the pressure rise across the pump as

$$
(\text { PRISE })_{\text {pump }}=\rho_{\text {in }} \mathrm{gH}
$$

where $\rho_{\text {in }}$ is the density of coolant at pump inlet.

\subsubsection{Pump Speed Equation}

At any time during a transient, the change in rotational speed of the pump is obtained from the application of angular momentum balance to the shaft and rotating assembly (including impeller, rotor, flywheel).

The equation of motion is as follows:

$$
\text { I } \frac{d \Omega}{d t}=\tau_{m}-\tau_{\text {hyd }}-\tau_{\text {fr }}
$$

where $I$ is the moment of inertia of shaft, impeller and rotating elements inside the motor, $\tau_{m}$ is the applied motor torque (=0 during coastdown), $\tau_{\text {hyd }}$ is the hydraulic load torque due to fluid at the impeller, ${ }^{\tau} \mathrm{fr}$ is the frictional torque and $\Omega$ is the angular speed of the pump ( $\mathrm{rad} / \mathrm{s})$.

If design speed is provided in rpm, the equation can be rewritten as

$$
\frac{2 \pi I}{60} \frac{d N}{d t}=\tau_{m}-\tau_{h y d}-\tau_{f r}
$$

The moment of inertia is a user-input parameter and depends on size and 
design of impeller, shaft, etc. This parameter has a strong influence on rate of pump coastdown during the first few seconds of transient. The hydraulic torque is obtained from pump characteristics (see Section 3.1.1). This is the predominant part of the unbalanced torque during most of the transient (except at very low flow and speed conditions). Frictional torque (due to bearing losses, fluid friction etc.), though very small in the initial few seconds of the transient, does become important at low system flow rate and pump speeds, such as occurs in the pump coastdown to natural circulation transient. (See Appendix A for details on formulation).

\subsection{PUMP TANK (RESERVOIR)}

During a transient, the fluid conditions in the pump tank determine the inlet boundary condition to the impeller.

Mass conservation at the pump tank yields the equation describing the level of coolant free surface below the cover gas as

$$
A_{\text {res }} \frac{d}{d t}\left(\rho Z_{R}\right)=W_{\text {in }}-W_{\text {out }}
$$

where $A_{\text {res }}$ is the cross-sectional area of the pump tank, $W_{\text {in }}$ is the mass flow rate into the pump, $W_{\text {out }}$ is the mass flow rate out of the pump, $Z_{R}$ is the height of coolant in the pump tank and $\rho$ is the density of coolant in the pump tank.

Assuming negligible gas flow to and from the cover gas volume in the reservoir and applying ideal gas law, the pressure of cover gas is determined from

$$
P_{\text {gas }}=\frac{m_{\text {gas }} R_{\text {gas }} T_{\text {gas }}}{A_{\text {res }}\left(Z_{\text {tot }}-Z_{R}\right)}
$$

where $Z_{\text {tot }}$ is the total height of the pump tank. The temperature of cover gas

$$
\div 14-
$$


is assumed to be the same as that of the coolant below it.

The inlet pressure to the pump impeller is governed by the behavior of coolant and cover gas in the pump tank, as follows:

$$
P_{\text {in }}=P_{\text {gas }}+\rho g Z_{R}+K_{\text {res }}\left(W_{\text {in }}-W_{\text {out }}\right)\left|\left(W_{\text {in }}-W_{\text {out }}\right)\right|
$$

where $K_{\text {res }}$ is a user-specified loss coefficient for the tank orifice. Knowing

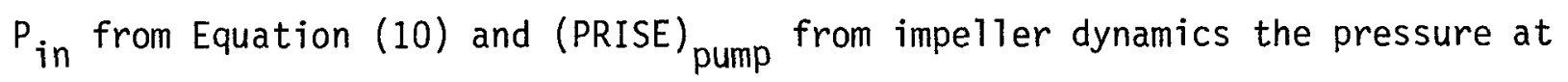
pump discharge is simply obtained as the sum

$$
P_{\text {out }}=P_{\text {in }}+(P R I S E)_{\text {pump }}
$$

\subsection{STEADY-STATE MODEL}

The aim of the steady-state pump mode1 is two-fold

(1) To determine the pump operating conditions from its characteristics, and

(2) To determine the level of sodium in the pump tank.

Impeller

In the current approach, it was felt more logical to determine pump operating speed during steady state by matching pump head with load (total hydraulic resistance) in the circuit. Thus, for the primary loop, the required pressure rise across the pump is obtained from:

$$
(\text { PRISE) })_{\text {pump }}=\sum_{j} \Delta P_{j}+\Delta P_{C V}+\Delta P_{R V}
$$

where $\Delta \mathrm{P}_{\mathrm{j} \text { 's }}$ are the pressure drops in the piping runs and the IHX. $\Delta \mathrm{P}_{\mathrm{CV}}$ and 
$\Delta \mathrm{P}_{\mathrm{RV}}$ are, respectively, the pressure drops in the check valve and the reactor vesse1.

For the intermediate loop

$$
\left(\text { PRISE }_{\text {pump }}=\sum_{j} \Delta P_{j}+\Delta P_{I H X, s}+\Delta P_{S H}+\Delta P_{E V}\right.
$$

where $\Delta P_{j ' s}$ are the pressure drops in the piping runs (not including $I H X$ ), $\Delta P_{S H}$, $\Delta P_{E V}$ are the shell side pressure drops in the superheater and evaporator, respectively, and $\Delta P_{I H X, S}$ is the secondary side pressure drop in the IHX.

Pump head is related to (PRISE) pump $_{\text {by: }}$

$$
H=(\text { PRISE })_{\text {pump }} /\left(\rho_{i n} g\right)
$$

Knowing the head and flow rate, the pump operating speed can be obtained from the homologous head curve. Equation (3) can be rearranged to give:

$$
a_{1} \alpha^{5}+a_{2} v \alpha^{4}+\left(a_{3} v^{2}-h\right) \alpha^{3}+a_{4} v^{3} \alpha^{2}+a_{5} v^{4} \alpha+a_{6} v^{5}=0
$$

This is a fifth degree polynomial equation in $\alpha$, and is solved by a Newton' iteration method specially adapted for polynomials. (8) Pump steady-state operating speed can now be obtained from Equation (2) since both $\alpha$ and $\Omega_{R}$ are known.

\section{Pump Tank}

The cover gas pressure in the tank at steady state is assumed known. For the primary loop, the gas pressure is generally equal to the gas pressure in the reactor vesse1. In the case of the secondary loop, the gas pressure is 
generally equal to that in the surge tank. Thus, static pressure balance yields the height of coolant in the pump tank:

$$
Z_{R}=\left(P_{i n^{-}}-P_{\text {gas }}\right) /\left(\rho_{i n^{g}}\right)
$$

where $\mathrm{P}_{\text {in }}, \rho_{\text {in }}$ are, respectively, the pressure and coolant density at pump inlet.

\subsection{PONY MOTOR OPTION}

While the arrangement of the primary and intermediate loops is intended to be such that natural circulation will provide sufficient heat transport for safe decay heat removal in the event of loss of all pumping power, the pumps may be equipped with pony motors (as is the case with CRBRP and FFTF) supplied with normal and emergency power to provide forced circulation decay heat removal. Therefore, an option for pony motor has been included in the pump model. The

pony motor is assumed to come into play when the main motor coasts down to a user-specified fraction of its rated speed. Once the pony motor takes over, the angular momentum balance equation i.e., Equation (6) is bypassed since pump speed remains constant. All other calculations remain the same.

\subsection{SYSTEM THERMAL-HYDRAULICS}

To test the pump model, it has to be coupled to the rest of the system. In our discussions here, we have focused attention on the primary system due to the more direct impact it has on core flow. Modeling for thermal-hydraulics in the intermediate system is done similarly.

Figure 5 illustrates an example configuration of the primary heat transport system for two-loop simulation of a three-loop plant, with a pipe-break 


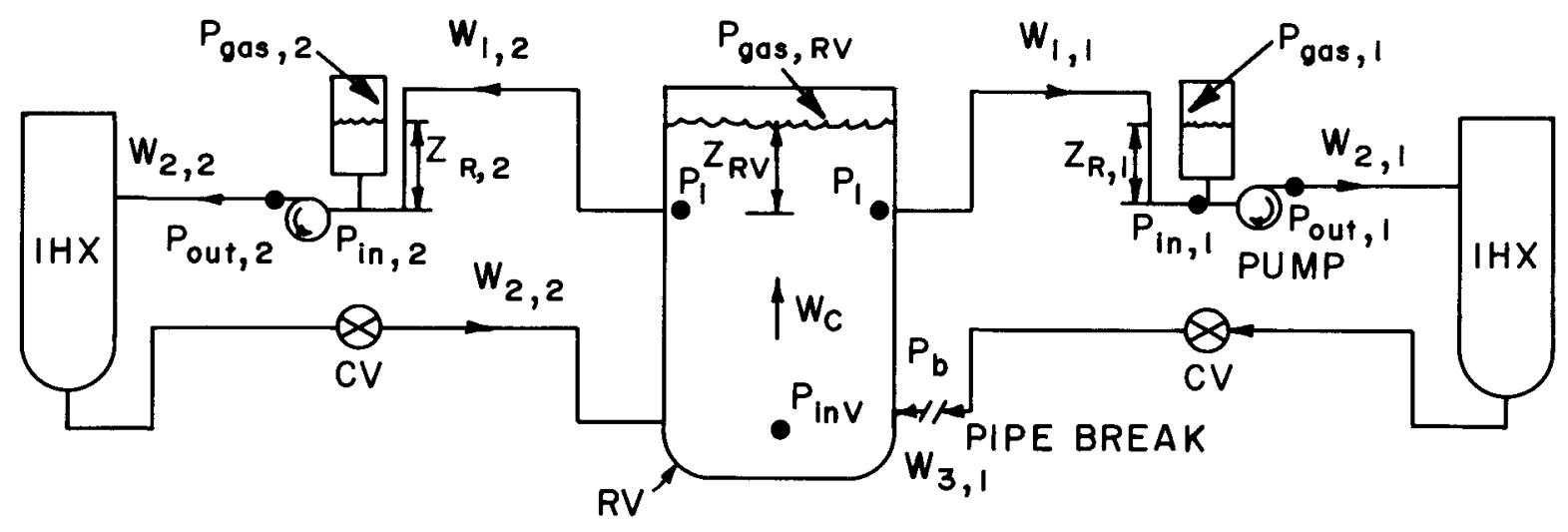

INTACT LOOPS

DAMAGED LOOP

Figure 5. Primary system configuration for twoloop simulation. 
near the reactor vessel inlet in one of the loops, and all intact loops being lumped together as the other loop. The pumps are shown located in the hot leg. This corresponds to the CRBR (or FFTF) primary loop design. There is one pump for each primary heat transport loop. For a pump coastdown transient, one-loop simulation with all three loops being lumped together is sufficient. The configuration would look much like Figure 5 without the broken loop.

The transient flow rates in different uniform mass flow rate sections of the loop are defined by volume-averaged momentum equations of the form

$$
\frac{d W}{d t} \sum \frac{L}{A}=P_{\text {in }}-P_{\text {out }}-\sum \Delta P_{f, g}
$$

where $\sum \frac{L}{A}$ is the inertia of fluid in the loop section, and $P_{\text {in }}$ and $P_{\text {out }}$ are the inlet and outlet pressures, respectively, for the loop section. For the section downstream of pump, $P_{\text {in }}$ represents the pump outlet pressure, and is obtained by evaluating pump inlet pressure from reservoir calculations and

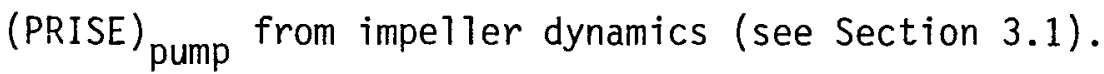

$\sum \Delta \mathrm{P}_{f, g}$ in Equation (17) is the sum of losses in all pipes and/or components in the section. For each pipe, the losses are defined by

$$
\Delta P_{f, g}=\Delta P_{f r}+\Delta P_{g}+\Delta P_{a c c}+\Delta P_{\text {other }}
$$

Here, $\Delta P_{f r}$ represents losses due to friction. $\Delta P_{g}$, the gravitational loss term (negative loss represents positive gravitational head), is dependent on system hydraulic configuration (elevations) and sodium density distribution. The density of sodium at any location is a function of temperature and is evaluated from loop thermal calculations. For details of general assumptions, model equations and solution methods for the system, the interested reader is referred to an earlier report on modeling for SSC-L. (1) 


\section{SAMPLE RESULTS}

Calculated results were obtained for the following two test cases:

1) Pump coastdown to natural circulation with reactor scram

2) Double-ended pipe break in the primary heat transport system (PHTS). For both cases, the plant is assumed operating at full power and rated thermalhydraulic conditions prior to the transient. Primary pump parameters used for the test cases are listed in Table I. These numbers are for the current design of

CRBRP. (9) For simplicity of presentation, system design and process parameters have not been 7 isted. The interested reader can obtain this information from a sample problem description in the users' manual for SSC-L.

\subsection{PUMP COASTDOWN WITH REACTOR SCRAM}

For this test case, a11 pumps in the heat transport system were tripped simultaneously at time zero, followed by reactor scram 0.75 seconds later. Pony motors were assumed inoperative due to loss of all power. Following loss of motor torque, the pumps start coasting down, causing the head across the impeller and the sodium flow rate in the loops to decrease. Figures 6 to 8 show predicted primary pump speed, flow and head transients resulting from such an accident, using the model equations described earlier along with equations for the rest of the system. Coastdown of pump speed is illustrated in Figure 6 . About 60 seconds into the transient, the curve shows a slight dip, indicating a more rapid rate of speed decay. This is due to the increased frictional losses in the pump that become dominant under low speed and flow conditions (see Appen$\operatorname{dix} A)$. Soon thereafter, at $(t=62 \mathrm{sec})$, the impeller comes to a complete standstill and then remains locked for the rest of the transient. The pump-to-vessel flow rate (see Figure 7 ) decays very similarly to the speed, but instead of 
TABLE I

Primary Pump Design Data Used in the Analysis

\begin{tabular}{|c|cc|}
\hline PARAMETER & VALUE \\
\hline$N_{R}$ & 1150 & $R P M$ \\
$Q_{R}$ & 2.1135 & $\mathrm{~m}^{3} / \mathrm{s}$ \\
$H_{R}$ & 137.16 & $\mathrm{~m}$ \\
$\tau_{R}$ & 26,927 & $\mathrm{~N}-\mathrm{m}$ \\
${ }^{\top}$ fr, $R$ & 0.035 & ${ }^{\mathrm{T}} \mathrm{R}$ \\
$I$ & 1180 & $\mathrm{~kg}-\mathrm{m}^{2}$ \\
$A_{\text {res }}$ & 3.0 & $\mathrm{~m}^{2}$ \\
$Z_{\text {tot }}$ & 6.0 & $\mathrm{~m}$ \\
$\rho_{\text {in, }}$ & 821.0 & $\mathrm{~kg} / \mathrm{m}^{3}$ \\
\hline
\end{tabular}




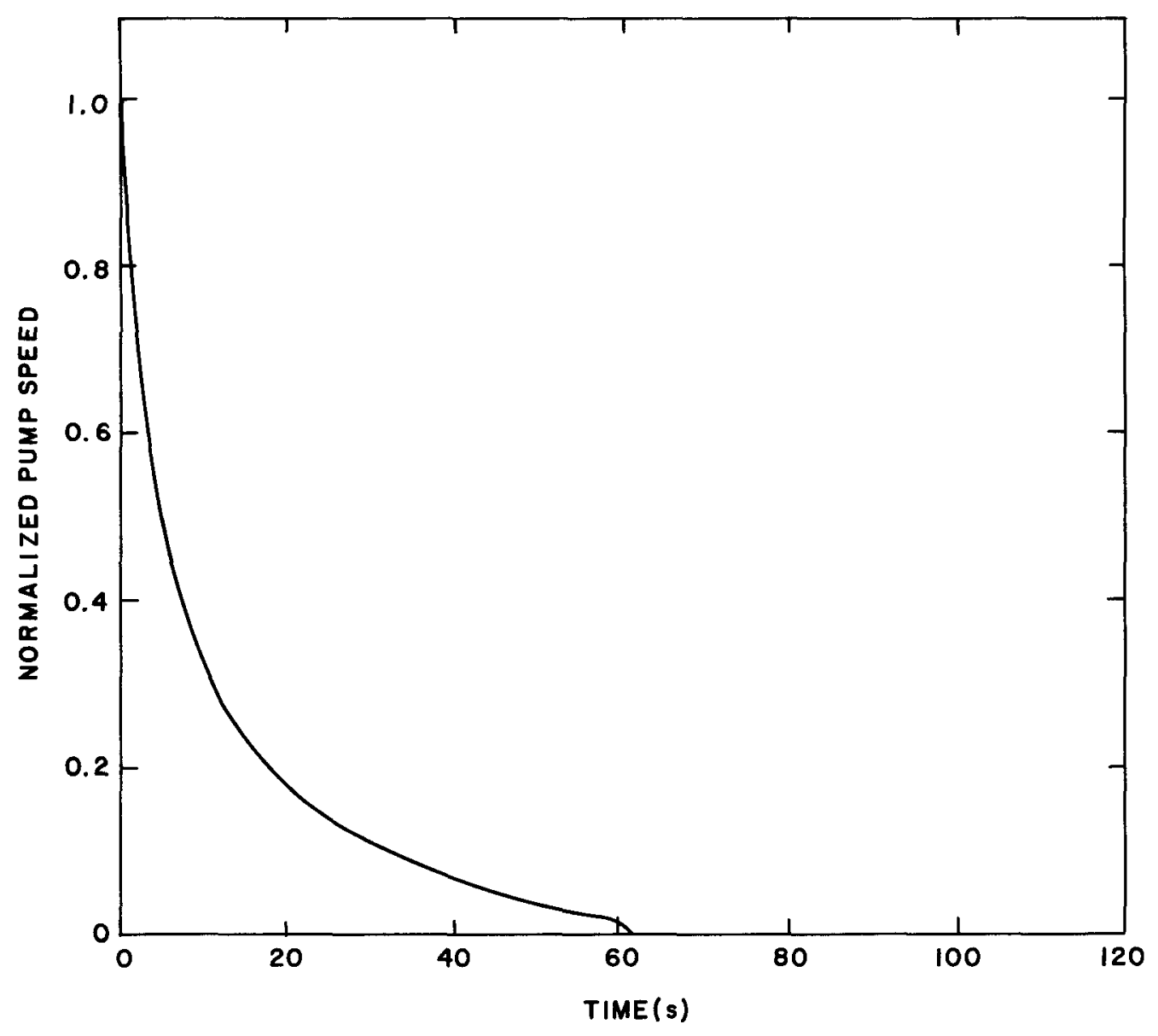

Figure 6. Computed primary pump speed decay for Case 1: pump coastdown with reactor scram. 
dropping to zero, it reaches a minimum of $3.4 \%$ of ful1 flow at $t \simeq 75 \mathrm{sec}$, after which it actually begins to show a slight increase. This is due to buoyancy effects in the system. Pump head, on the other hand, drops very rapidly in the first few seconds of transient, and with pump speed approaching zero, it becomes negative, representing the pump condition as a resistance to flow in the circuit. This is not evident in Figure 8 due to its scale. The transient operating points of the impeller are shown plotted on its homologous head characteristics in Figure 9. Here, the condition of negative head is very clearly evident for $t \geq 58 \mathrm{sec}$.

In order to study the effect of pump friction on predicted coastdown, two different correlations were applied in turn to the pump model:

1) The RELAP3B ${ }^{(7)}$ pump friction equation, given by

$$
\tau_{f r}=\tau_{f r, R} \alpha|\alpha|
$$

where $\tau_{f r, R}$ is the frictional torque at rated conditions.

2) The DEMO ${ }^{(11)}$ pump friction formulation (see Appendix A).

Comparison of predicted results using these two formulations are illustrated in Figure 10. Figure 10(a) shows pump speed decay. Initially, since pump rotational inertia is the dominating influence governing the coastdown, no noticeable difference in predictions is observed. As low pump speeds, however, the choice of correlation is seen to have a dramatic effect on the results. The DEMO formulation predicts pump standstill to occur at $t \simeq 62 \mathrm{sec}$, whereas with the RELAP equation, the impeller is still rotating with $4 \%$ of rated speed at $t=120 \mathrm{sec}$. Figure $10(\mathrm{~b})$ shows the effect on predicted flow transients. With the DEMO friction formulation, natural circulation is established in the system at $t \simeq 75 \mathrm{sec}$. With the RELAP equation, while predicted flow rate is in general 


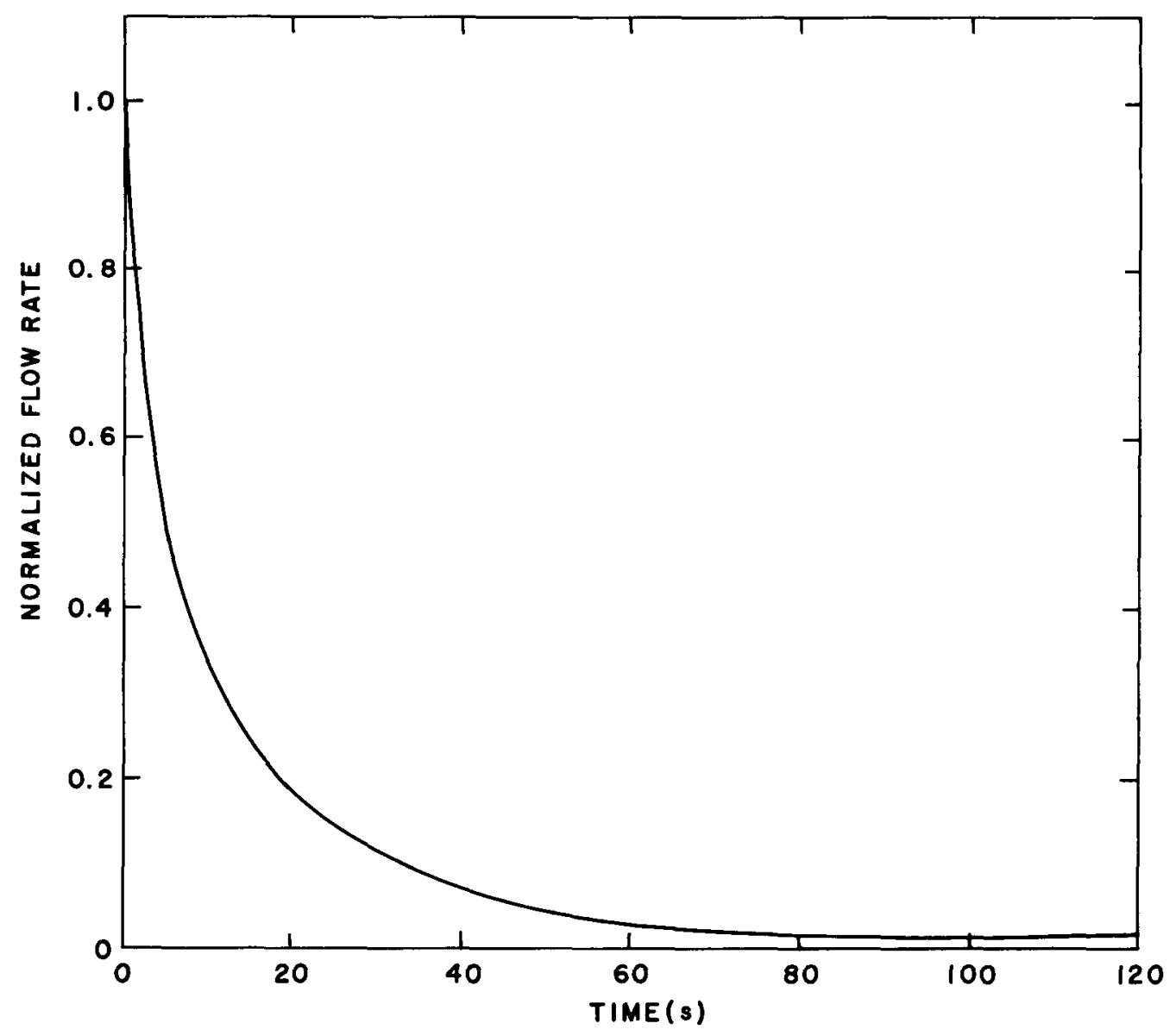

Figure 7. Computed pump-to-vessel flow decay for Case 1. 


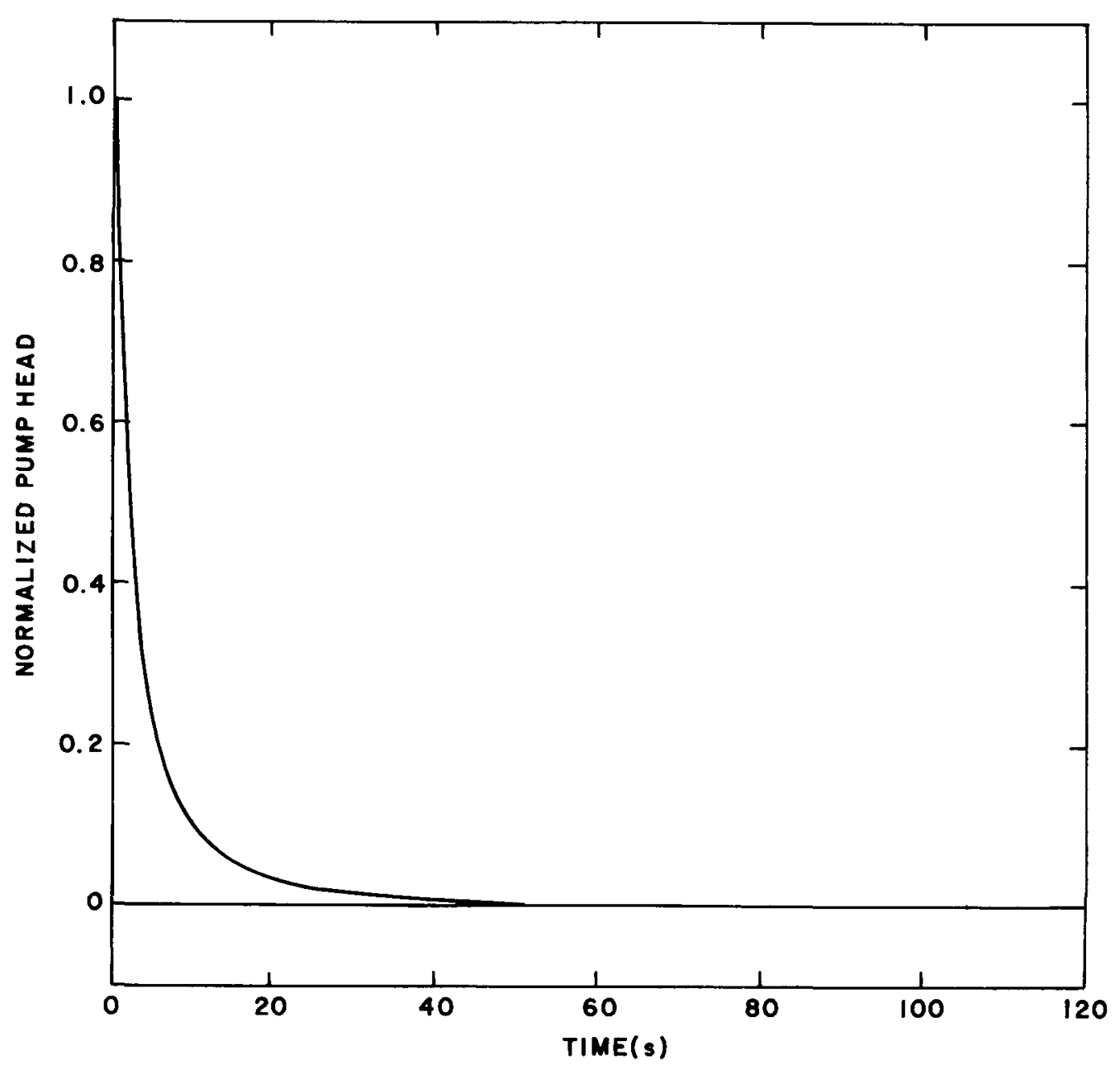

Figure 8. Computed decay of primary pump head for Case 1 . 


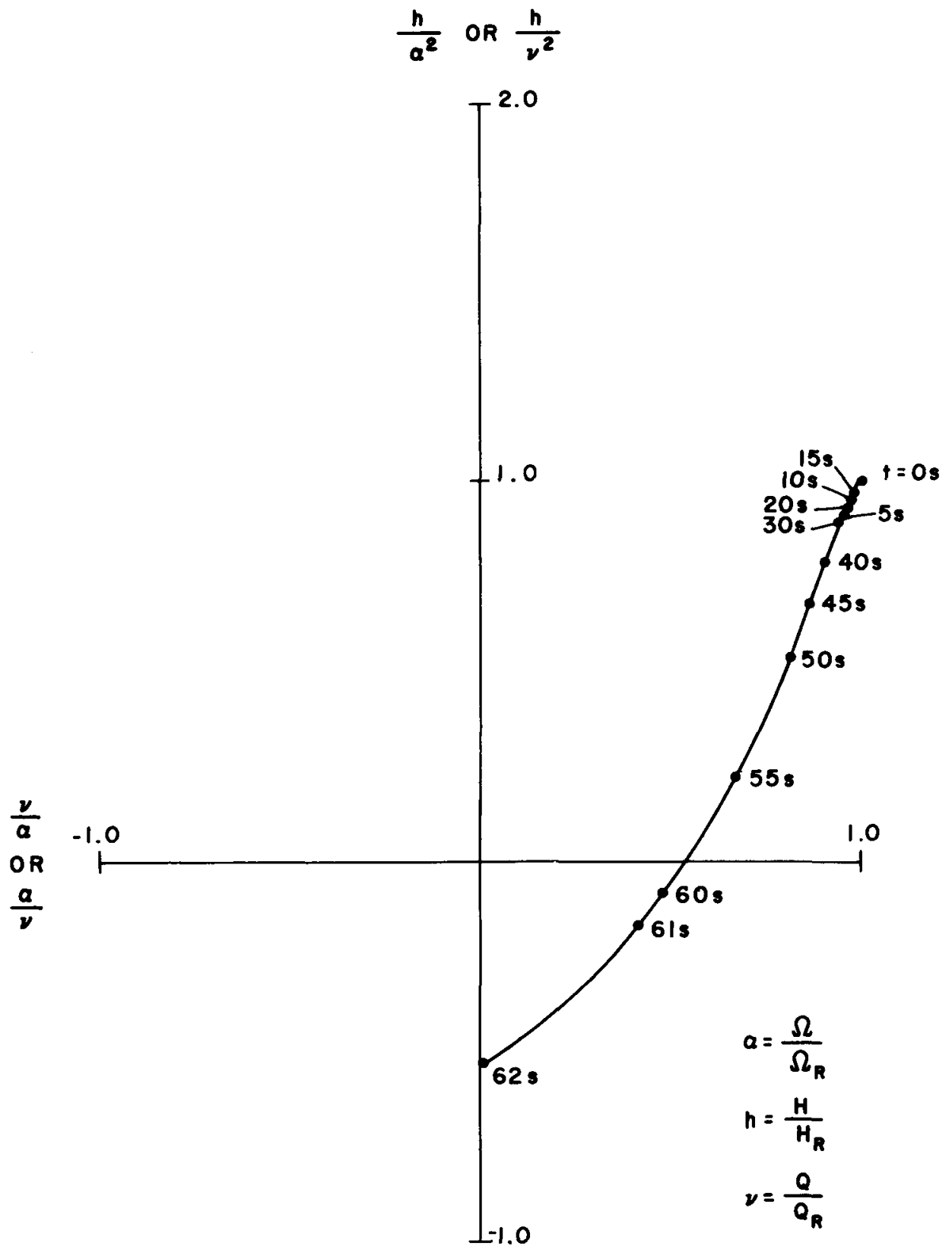

Figure 9. Transient operating points on pump head curves for Case 1 . 


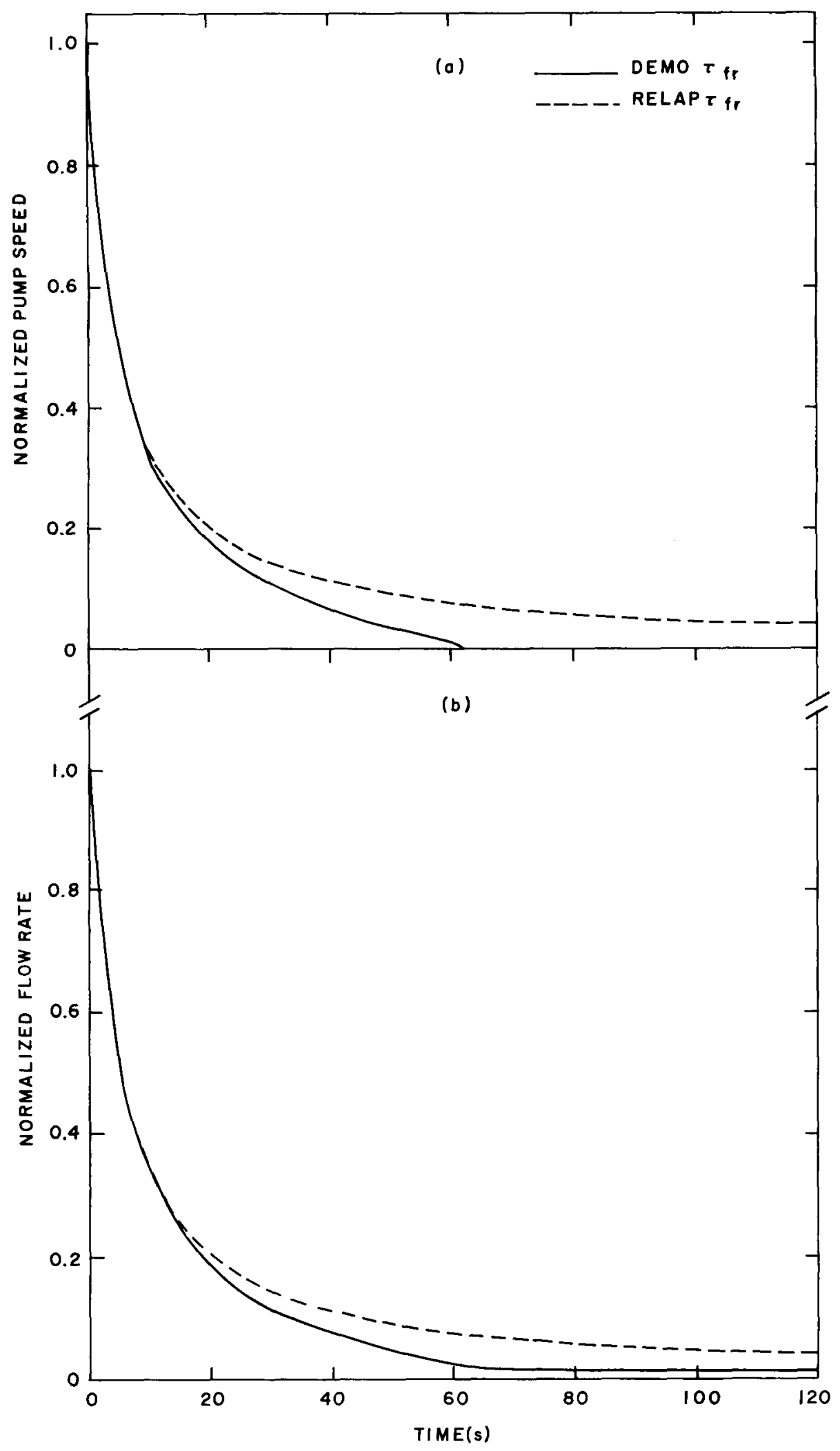

Figure 10. Comparison of predicted pump speed and flow decay for Case 1 using DEMO and RELAP3B pump friction equations. 


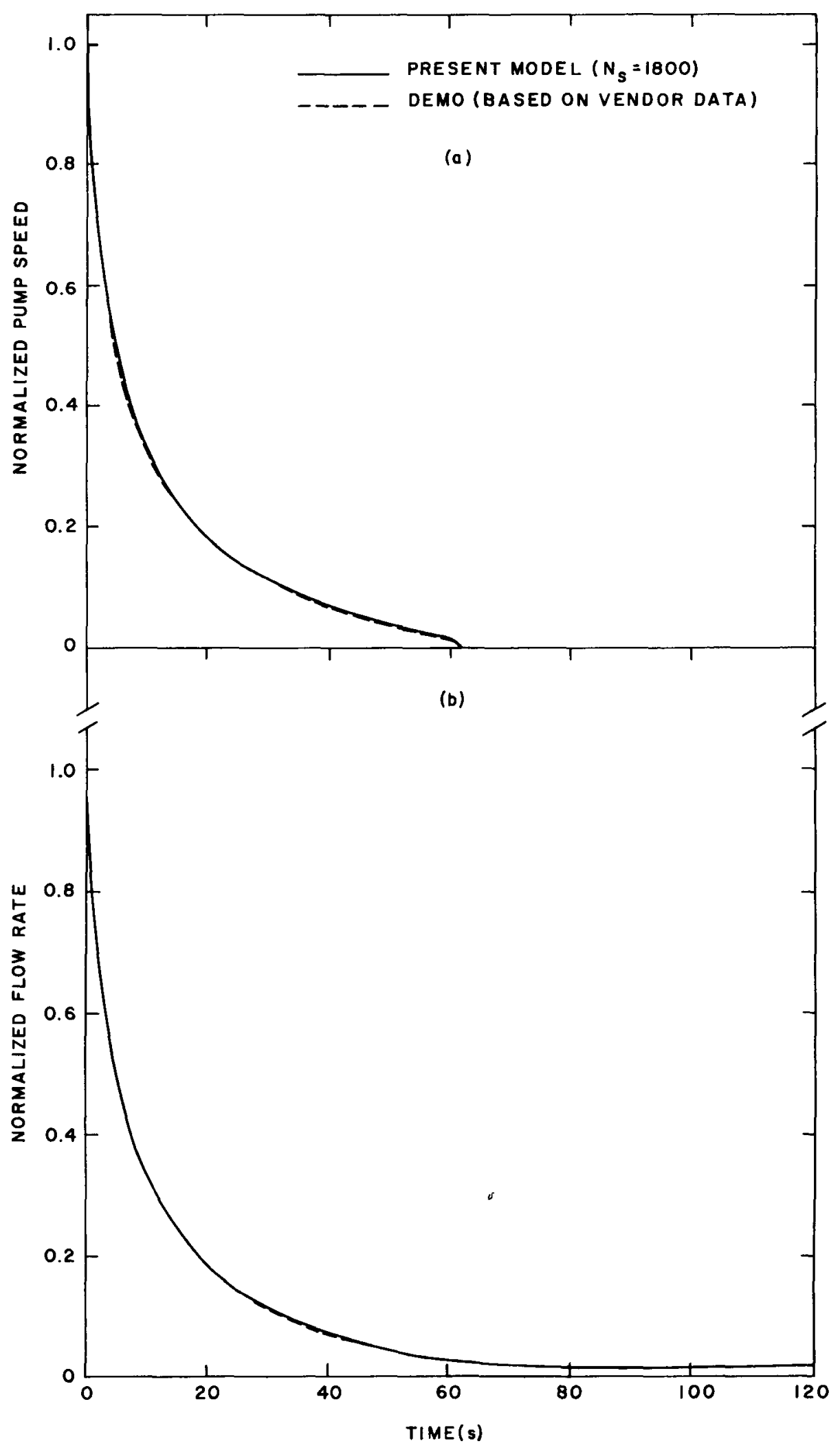

Figure 11. Comparison of predicted speed and flow transients for Case $I$ using pump characteristics from DEMO and present model. 
higher, it is still decaying even at $t=120 \mathrm{sec}$.

As mentioned in the previous chapter, the homologous characteristics for a11 regions of operation have been derived from test results for a model pump with $N_{S}=1800$. In Figure 11, we can see comparisons of predicted coastdown using the characteristic curves in DEMO ${ }^{(11)}$ (based on vendor data for CRBR pumps in the zone of normal operation) and the curves in the present model. The results are seen to agree very closely. This indicates that the characteristics from the model pump with $N_{S}=1800$ are applicable for analysis of the CRBR pump with $\mathrm{N}_{\mathrm{S}}=2200$.

\subsection{DOUBLE-ENDED PIPE BREAK IN THE PHTS}

For this test case, a double-ended break with $A_{b}=A_{p}$ was postulated to occur in the primary cold leg piping near the reactor vessel inlet. Guard vessel was assumed present, and primary cold leg check valves were assumed to be stuck open in failed position. Results for this test case were obtained with a stand alone code for the heat transport system, together with simplified representation of core hydraulics. Hence, even though important qualitatively, the calculated response need not represent the system response that would obtain with a more detailed reactor hydraulic model included.

In looking at the primary 10op configuration of Figure 5, one would guess that pumps in the intact loops, being more remote from the break, will experience transients less severe although generally similar in nature compared to the pump in the broken loop. This conclusion can be more clearly drawn by examining Figure 12, where computed flow, speed and head transients for both broken and intact loop pumps are illustrated. During the first half-second following break, the pump discharge in the broken loop is seen to increase sharply above its steady state value. This is because of the sudden drop in 


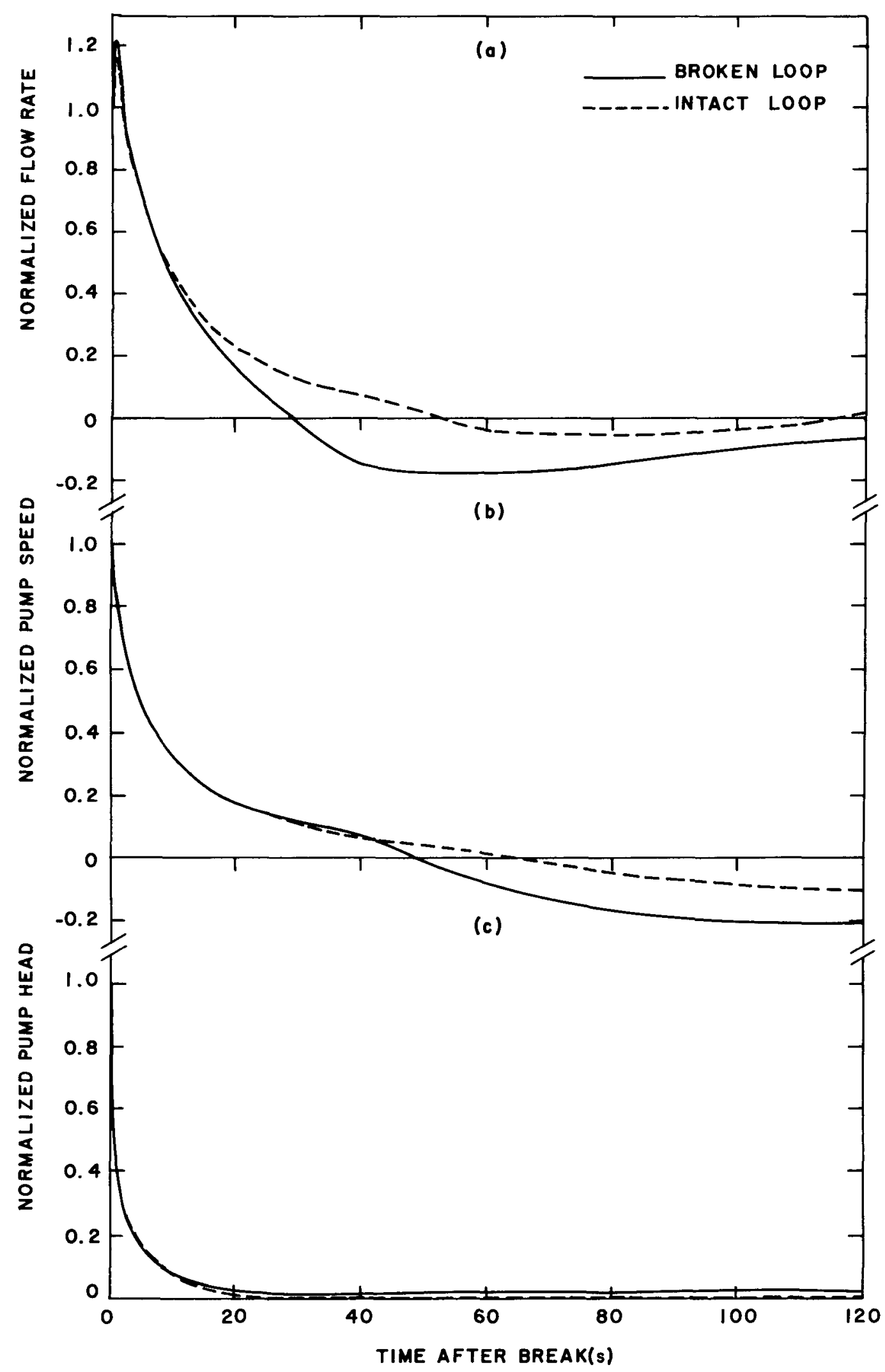

Figure 12. Computed pump flow, speed and head transients in broken and intact loops for Case 2: double-ended pipe break in the PHTS. 
flow resistance following break. In the intact loops, the pump-to-vessel flow also shows an increase, but not as sharp as in the broken loop. Following that, for the first 10 to 12 seconds, the flow decay is very similar for all loops, with broken loop flow going out the break and spilling into the guard vessel, and intact loop flow dividing itself between flow through core and flow out of break. Once the guard vessel fills up and stops further break flow, the intact loop flow attains communication with broken loop pump, causing rapid flow decay leading to flow reversal in the rest of the broken loop. The flow in intact loops reverses much later and eventually, at around 115 seconds, even recovers and becomes positive again. During the first 20 seconds, the inertia dominated speed coastdown is very similar for pumps in both loops. However, early flow reversal in the broken loop causes early reversal of pump speed in that loop, as shown in Figure 12(b).

It is interesting to see that even with the surge of flow increase during the first half-second following break, the pumps do not overspeed. This is in quite a contrast to the case in 1 ight water reactors, where pump overspeed is an important concern in pump transient analysis. The contributing factor for this difference in response is the low system pressures for LMFBRs as compared to 1 ight water reactors, resulting in gradual system depressurization as opposed to drastic depressurization following pipe break in the primary loop.

Pump head [Figure 12(c)] is seen to decay similarly for both intact and broken loops, with intact loop pumps providing a slightly higher head. Later in the transient when flow reversal takes place, pump head is seen to remain positive. This is because head is defined by

$$
H=\left(P_{\text {out }}-P_{\text {in }}\right) / \rho_{\text {in }} g
$$


and on flow reversal, since flow is entering through the discharge line and exiting through the suction line, $P_{\text {out }}$ is always going to be larger than $P_{\text {in }}$ due to pressure losses in the pump. The higher head for the broken loop pump merely points to the larger negative flow in the loop.

The transient results of Figure 12 can be collapsed into the homologous head curves of Figures 13 and 14. As an example, reference is made to Figure 13, which shows transient operating points for the broken loop pump. Initially, speed falls off more rapidly than flow due to the larger inertia of fluid as compared to pump inertia, and the operating points progress downward along the HVN curve. Soon, the trend is reversed and the pump operation moves up along HVN, until, at $t=20 \mathrm{sec}, \alpha=\nu$. For $t>20 \mathrm{sec}, \alpha$ becomes greater than $v$, i.e., $\left|\frac{v}{\alpha}\right|<1$, and the transient moves into HAN curve. With flow continuing to decay, the operating points progress along HAN until flow reverses, and then they enter the HAD curve. Following flow reversal, pump speed starts decaying more rapidly. The combined effect of decreasing positive pump speed and increasing negative flow rate causes $\left|\frac{v}{\alpha}\right|$ to exceed 1 , and the transient moves into HVD. At $t \simeq 50 \mathrm{sec}$, pump speed also reverses, and pump operation enters the HVT curve. Once in the turbine region, the speed continues to increase in the negative direction until, at $t \simeq 76 \mathrm{sec},\left|\frac{v}{\alpha}\right|$ is again less than 1 , and the pump enters the HAT curve.

The intact loop pump travels more slowly through its characteristics so that at $52 \mathrm{sec}$, it is still in normal operation zone. Once flow reverses in the intact loop, however, it travels more rapidly along its characteristics. In fact, it even enters the reverse pump region when at $t \simeq 115 \mathrm{sec}$, the flow in intact loop recovers.

These results, besides illustrating the nature of transients experienced 
by the broken and intact loop pumps, also bring out the necessity of representing all regions of operation in the pump characteristics. 


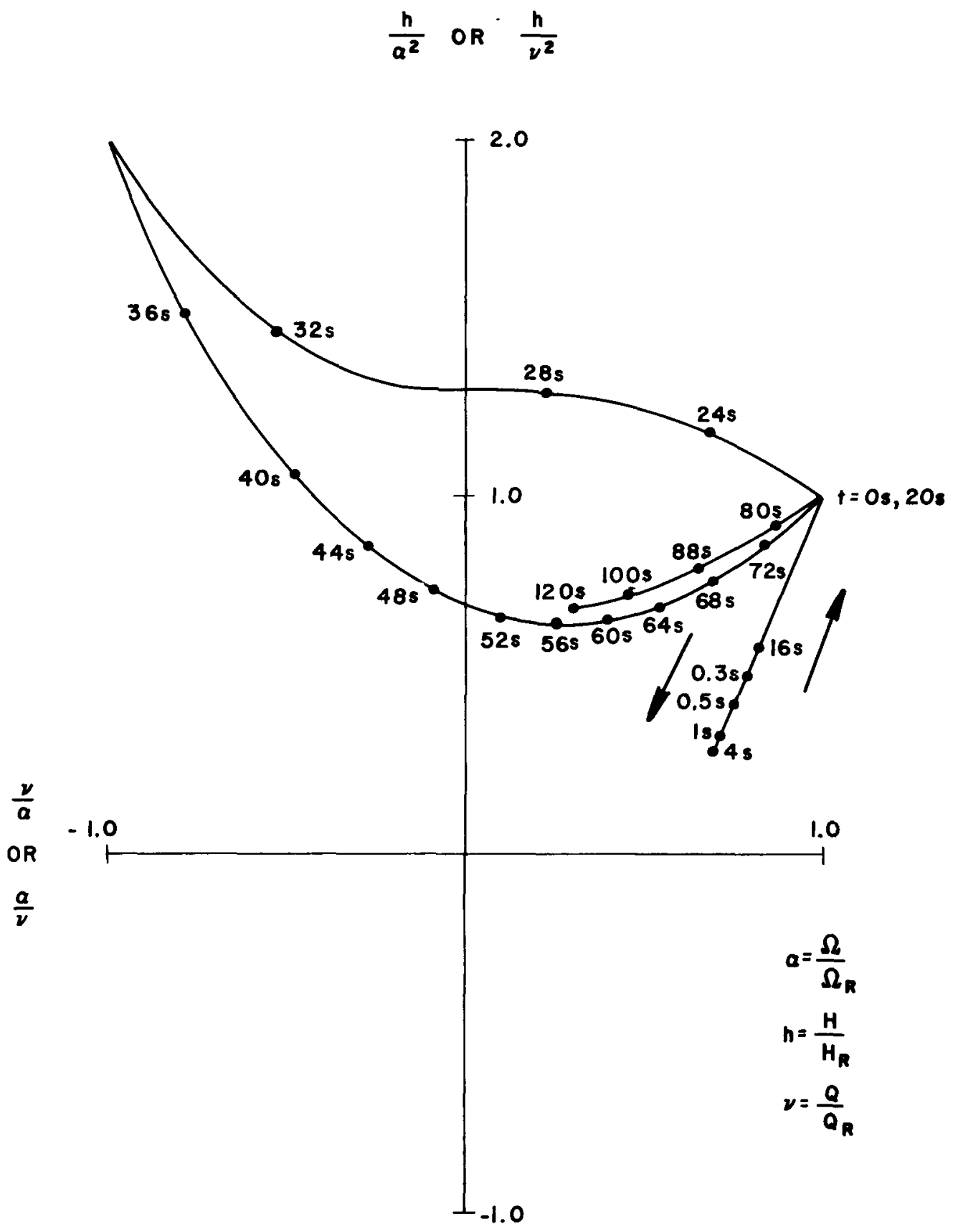

Figure 13. Transient operating points on homologous head curves for broken loop pump. 


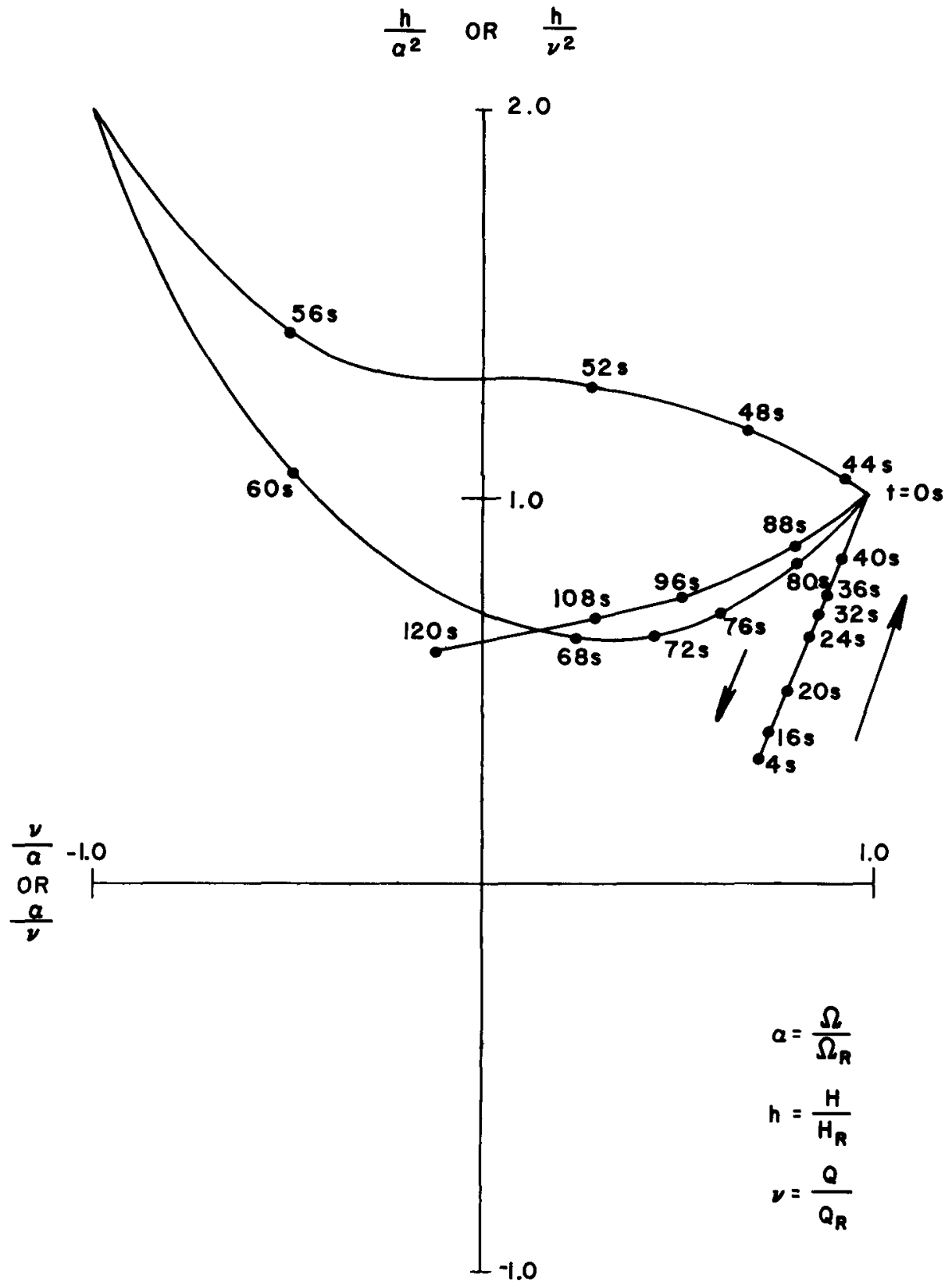

Figure 14. Transient operating points on homologous head curves for intact loop pump. 


\section{DISCUSSION}

A single-phase pump model for analys is of system transients in LMFBR heat transport systems has been presented where homologous characteristic curves are used to predict the behavior of the turbomachine during operating transients. The model which has been developed for analysis of pumps in loop-type LMFBRs, should be applicable, with minimal alterations, to represent pump behavior in pool-type LMFBRs as wel1. From calculated results for the two test cases, the following inferences are derived:

1) Representation of pump characteristics for all regions of operation, as is done here, forms an essential part of the pump model.

2) Homologous characteristics for pumps in the same range of specific speeds (1800 for model v/s 2200 for prototype) gave results that agreed very closely. This provides more credibility to the application of model test results with similar pumps to analysis of full-scale units.

3) Frictional losses in the pump become very important under low flow and speed conditions. Hence, the selection of correlation for frictional torque will influence the predicted onset of natural circulation in the system.

For future work, it will be necessary to study the effects of cavitation on pump performance during severe transients such as would ensue following a double-ended pipe break in the primary system. Also, for simulation of startup and load following transients, it will be necessary to include modeling for the drive motor torque, coupled to the model for plant control system. A model for cover gas flow into and out of the pump tank may be required to accurately simulate transients from a break in the vicinity of the pump. Experimental 
data is needed on frictional losses in liquid metal pumps as a function of flow and speed, both in the forward and reverse direction. Finally, even though there is evidence $(14,15)$ to support the use of quasi-steady pump performance data to analyze transient pump performance, it may be worthwhile to experimentally investigate the limits of applicability of this procedure. 


\section{APPENDIX A}

\section{A.1 Pump Performance Curves}

The performance data from Streeter and Wylie ${ }^{(3)}$ and Donsky ${ }^{(4)}$ for a centrifugal pump with $\mathrm{N}_{\mathrm{S}}=1800$ (gpm units) were fitted with polynomials for each operational region. When possible, an attempt was made to fit two adjacent regions with the same curve. Since most of the data are quoted with 3 significant digits, an arbitrary deviation of one part in a thousand was assigned to each data point. Then, defining a convergence criterion

$$
x^{2}=\sqrt{\sum_{k=1}^{N P} \frac{\left(y_{d a t a}^{k}-y_{c o m p}^{k}\right)^{2}}{\left(0.001 y_{d a t a}^{k}\right)^{2}}}=\text { minimum }
$$

a least square, random search and gradient search procedure ${ }^{(13)}$ was applied to determine the coefficients of the polynomials

$$
y_{\text {comp }}^{k}=\sum_{i=1}^{M} c_{i} x_{k, \text { data }}^{i}(3 \leq M \leq 8, k=1, N P)
$$

where NP is the number of data points and $M$ is the order of the polynomial. For all the fits with $4 \leq M \leq 7$ the $x^{2}$ reached $0(10)$ or better for each point, thus ensuring a lower limit accuracy of $1 \%$ for reproducibility of the data through the analytical curves. However, $M=6$ was found to give the best overa11 accuracy. Finally, these curves were graphically reproduced in order to have a visual check for unrealistic or undesirable inflections between data points, and it was felt that $M=6$ would ensure smoothness for all regions except reverse pump, where the limited number of data points available caused smoother fits with lower order polynomials. In this way, seven head curves and seven torque curves have been defined to represent pump characteristics 
through all operational regions with an accuracy of around $1 \%$ or better.

Head

The polynomial equations are of the form

$$
\begin{aligned}
\frac{h}{\alpha^{2}}=c_{0}+c_{1} \frac{\nu}{\alpha}+c_{2}\left(\frac{\nu}{\alpha}\right)^{2}+ & c_{3}\left(\frac{v}{\alpha}\right)^{3}+c_{4}\left(\frac{v}{\alpha}\right)^{4}+c_{5}\left(\frac{v}{\alpha}\right)^{5} \\
& \text { for HAN, HAD, HAT, HAR }
\end{aligned}
$$

and

$$
\begin{array}{r}
\frac{h}{\nu^{2}}=c_{0}+c_{1} \frac{\alpha}{v}+c_{2}\left(\frac{\alpha}{v}\right)^{2}+ \\
+c_{3}\left(\frac{\alpha}{v}\right)^{3}+c_{4}\left(\frac{\alpha}{v}\right)^{4}+c_{5}\left(\frac{\alpha}{v}\right)^{5} \\
\text { for HVN, HVD, HVT, HVR }
\end{array}
$$

The values of the coefficients built-in for all regions are listed in Table A.I.

\section{Torque}

The polynomial equations are of the form

$$
\begin{aligned}
\frac{\beta}{\alpha}=c_{0}+c_{1} \frac{\nu}{\alpha}+c_{2}\left(\frac{\nu}{\alpha}\right)^{2}+ & c_{3}\left(\frac{v}{\alpha}\right)^{3}+c_{4}\left(\frac{\nu}{\alpha}\right)^{4}+c_{5}\left(\frac{\nu}{\alpha}\right)^{5} \\
& \text { for BAN, BAD, BAT, BAR }
\end{aligned}
$$

and

$$
\begin{array}{r}
\frac{\beta}{v^{2}}=c_{0}+c_{1} \frac{\alpha}{v}+c_{2}\left(\frac{\alpha}{v}\right)^{2}+c_{3}\left(\frac{\alpha}{v}\right)^{3}+c_{4}\left(\frac{\alpha}{v}\right)^{4}+c_{5}\left(\frac{\alpha}{v}\right)^{5} \\
\text { for BVN, BVD, BVT, BVR }
\end{array}
$$

The values of the coefficients built-in for all regions are listed in Table A.II. 
TABLE A.I

Head Polynomial Coefficients

\begin{tabular}{|c|c|c|c|c|c|c|c|}
\hline Curves & HVN & HAN + HAD & HVD & HVT & HAT & HAR & HVR \\
\hline$c_{0}$ & -0.558 & 1.2897 & 0.69189 & 0.69209 & 0.63405 & 0.63405 & -0.55600 \\
$c_{1}$ & 0.85376 & -0.061907 & 0.43961 & -0.46132 & 0.20178 & 0.14665 & 0.66362 \\
$c_{2}$ & 0.82906 & 0.17327 & 0.68459 & 0.92592 & -0.30242 & -4.1896 & -0.036081 \\
$c_{3}$ & -3.7106 & -0.57294 & -0.24701 & -0.4308 & 0.76603 & -2.4828 & -0.93928 \\
$c_{4}$ & 7.0593 & 0.033762 & 0.63156 & 0.50845 & -0.48077 & 0.89730 & -0.57381 \\
$c_{5}$ & -3.4776 & 1.3865 & -0.20833 & -0.22436 & 0.19231 & 0.0 & 0.0 \\
\hline
\end{tabular}


TABLE A.II

Torque Polynomial Coefficients

\begin{tabular}{|c|c|c|c|c|c|c|c|}
\hline Curves & BVN & BAN + BAD & BVD & BVT & BAT & BAR & BVR \\
\hline$c_{0}$ & -0.37069 & 0.44652 & 0.8658 & 0.86533 & -0.68468 & -0.684 & -0.372 \\
$c_{1}$ & 0.41741 & 0.5065 & 0.28437 & -0.60816 & 1.8495 & 2.0342 & 2.3716 \\
$c_{2}$ & 3.8511 & 0.59643 & -0.22348 & 3.1497 & 0.96871 & -0.95477 & -0.56147 \\
$c_{3}$ & -7.6752 & -0.64055 & 0.45083 & -4.3647 & -8.9653 & -0.42286 & 0.0 \\
$c_{4}$ & 7.0695 & -0.025531 & -0.70586 & 10.418 & 12.045 & 0.0 & 0.0 \\
$c_{5}$ & -2.2917 & 0.11531 & 0.21562 & -4.0064 & -4.7546 & 0.0 & 0.0 \\
\hline
\end{tabular}




\section{A.2 Pump Friction}

Torque due to frictional losses in the pump is represented in the model as polynomial equations of the form

$$
{ }^{\tau} f r=\tau_{R}\left(c_{0}+c_{1} \alpha+c_{2} \alpha|\alpha|\right)
$$

where $\tau_{R}$ is the rated hydraulic torque and $\alpha$ is the normalized pump speed.

At present, the DEMO equation (which has in large part been derived from pump vendor data for CRBRP pumps ${ }^{(12)}$ ), is used to provide the coefficients, except at very low speeds.

The coefficients in Equation (A-7) are

$$
\begin{array}{ll}
c_{0}=0.012, \quad c_{1}=0.023, \quad c_{2}=0.0 & \text { for } \alpha>0.0117 \\
c_{0}=0.117, \quad c_{1}=-8.97, \quad c_{2}=0.0 & \text { for } \alpha \leq 0.0117 .
\end{array}
$$

However, for very low speeds (approaching locked rotor), unrealistically high values of $\tau_{\mathrm{fr}}$ are obtained with the DEMO relations, so a third region is defined where

$$
c_{0}=0.0, c_{1}=14.77, c_{2}=0.0 \quad 0 \leq \alpha \leq 0.005 .
$$

This gives the correct limit for $\alpha=0$, and removes any instabilities that would be caused in the hydraulic integrator otherwise. 


\section{REFERENCES}

1. A. K. Agrawa1, et a1., "An Advanced Thermohydraulic Simulation Code for Transients in LMFBRs (SSC-L Code)," Brookhaven National Laboratory, BNLNUREG-50773 (February 1978).

2. N. J. Palladino, "Mechanical Design of Components for Reactor Systems," Ch. 14 in The Technology of Nuclear Reactor Safety, Vol. 12, edited by T. J. Thompson and J.G. Beckerley, The M.I.T. Press, Cambridge, Massachusetts (1973).

3. V. L. Streeter and E. B. Wylie, Hydraulic Transients, McGraw-Hil1, New York (1967).

4. B. Donsky, "Complete Pump Characteristics and the Effects of Specific Speeds on Hydraulic Transients," ASME Transactions, Journal of Basic Engineering, pp. 685-696 (December 1967).

5. A. J. Stepanoff, "Centrifugal and Axial Flow Pumps: Theory, Design and Application," 2nd Edition, John Wiley \& Sons, Inc. New York (1957).

6. P. W. Runstadler, Jr., "Review and Analysis of State-of-the-Art of Multiphase Pump Technology," Electric Power Research Institute, EPRI-159 (February 1976).

7. "RELAP3B Manua1, A Reactor System Transient Code," Brookhaven National Laboratory, RP 1035, (December 1974).

8. E. Isaacson and H. B. Keller, Analysis of Numerical Methods, John Wiley \& Sons, Inc., New York (1966).

9. Clinch River Breeder Reactor Project, Preliminary Safety Analysis Report, Project Management Corporation, (July 1975).

10. Agrawal, A. K., Cazzoli, E. G., Guppy, J. G., Kennett, R. J., Nepsee, T. C., and Srinivasan, E. S., Users' Manual for SSC-L code. Brookhaven National Laboratory Topical Report (in preparation).

11. LMFBR Demonstration Plant Simulation Model, DEMO," Westinghouse Advanced Reactors Division, WARD-D-0005, (Rev. 4), (January 1976).

12. A. Batenburg, Westinghouse Advanced Reactors Division, private communication, (March 1978).

13. F. James and M. Roos, "MINUTS - "A package of programs to minimize a function of at most 15 variabies, compute the covariance matrix and find the true errors," CERN Computer Center Program Library-D506.

14. R. T. Knapp, "Complete Characteristics of Centrifugal Pumps and their use in the Prediction of Transient Behavior," ASME Transactions, Vol. 59, pp. 683-689 (1937).

15. D. J. 01son, "Single- and Two-Phase Performance Characteristics of the MOD-1 Semiscale Pump Under Steady State and Transient Fluid Conditions," Aerojet Nuclear Company, ANCR-1165, VC-78d (October 1974). 